\title{
THE TENSILE STRENGTH OF POLYCRYSTALLINE ICE
}

\section{(Abstract only)*}

by

\author{
E. M. Schulson and J. H. Currier**
}

(Thayer School of Engineering, Dartmouth College, Hanover, New Hampshire 03755, U.S.A.)

\section{ABSTRACT}

Structure/property relationships, while wellresearched in metallic and in some ceramic materials, have been essentially ignored in studies on the mechanical properties of ice. To rectify this situation, experiments have been designed and have been underway for the past two years to investigate one mechanical property, i.e. tensile strength, and the effect of one structural feature, i.e. grain size, on this property. A clear relationship has been established, and is reported here. Other work is in progress and will also be reported in due course.

Equiaxed and randomly oriented aggregates of freshwater ice, of grain size (as seen in twodimensional sections) varying from 1.0 to $7.3 \mathrm{~mm}$, were prepared in the form of large cylinders $(91 \mathrm{~mm}$ diameter $\times 231 \mathrm{~mm}$ length). The aggregates were deformed to fracture under uniaxial tension, using a specially designed ball-joint and yoke assembly to ensure axial loading. Data were obtained at $-10 \pm 0.2^{\circ} \mathrm{C}$ (i.e. at $96 \%$ of the melting point) at a strain-rate of $10^{-6} \mathrm{~s}^{-1}$.

Figure 1 shows that the tensile strength decreases with increasing grain size, from $1.25 \mathrm{MPa}$ for $d=1 \mathrm{~mm}$ to $0.80 \mathrm{MPa}$ for $\mathrm{d}=7 \mathrm{~mm}$. Moreover, this figure illustrates that the data are highly reproducible; i.e. that strength is reproducible to within $\pm 5 \%$ for a given grain size over the complete range.

Concerning the functional relationship between tensile strength of and grain size, analysis shows that the following equation is well obeyed:

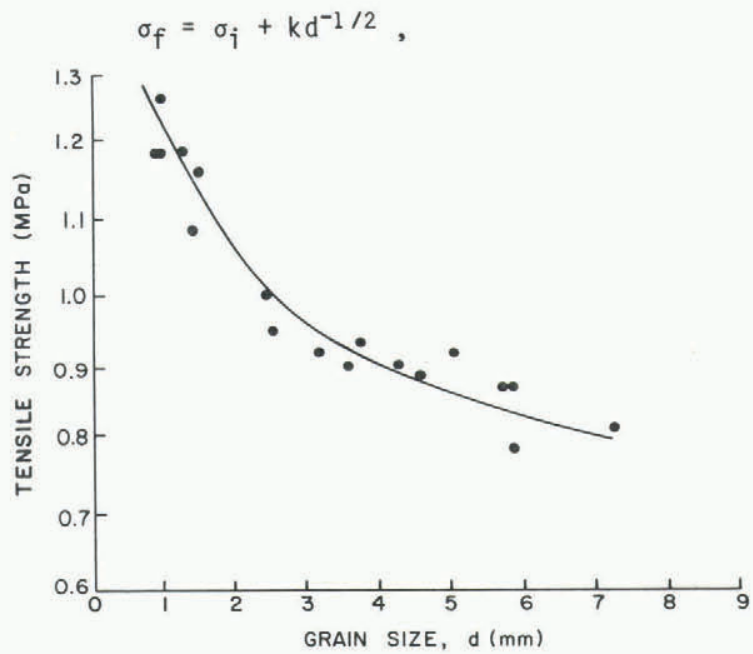

Fig.1. Graph showing the decrease in the tensile strength of ice with increasing grain size. where $\sigma_{j}$ is $0.6 \mathrm{MPa}$ and $\mathrm{k}$ is $0.02 \mathrm{MPa} \mathrm{m} \mathrm{m}^{1 / 2}$ at $-10^{\circ} \mathrm{C}$ and $10^{-6} \mathrm{~s}^{-1}$. This point is illustrated in Figure 2 .

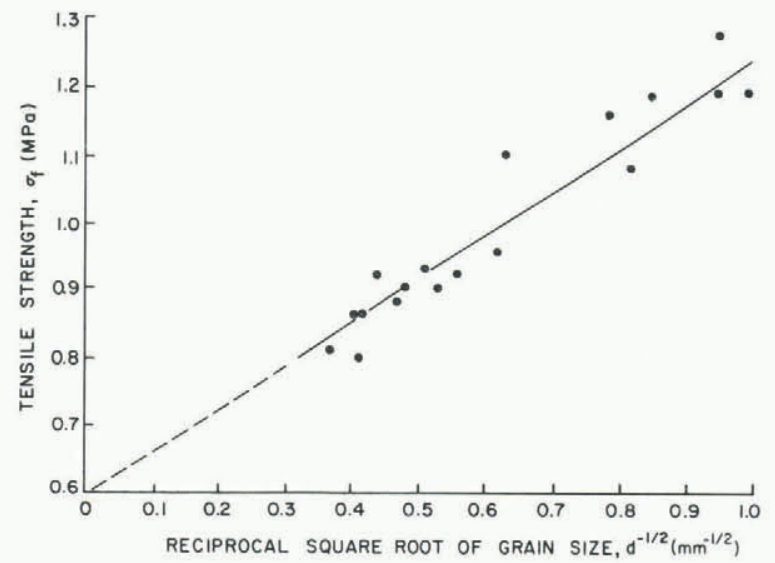

Fig.2. Hall-Petch plot showing the relationship between the tensile strength and the grain size of the ice.

The $d^{-1 / 2}$ character of this relationship, which is of the classical Hall-Petch form observed frequently in metallic materials, indicates that the tensile strength of ice is controlled by some process involving stress concentration, possibly the propagation of microcracks nucleated by the interactions of dislocations or the propagation of pre-existing defects. Of these, the foriner is the more probable. The reason is that processes involving dislocation motion, when expressed by the difference $\sigma_{f}-\sigma_{i}$, are expected to increase linearly with increasing $d^{-1 / 2}$, whereas processes involving the propagation of pre-existing defects predict a linear relationship between $\sigma_{f}$ and $d^{-1 / 2}$ which extrapolates through the origin. The former behavior is the one observed.

It is thus concluded: (i) that the tensile strength of equiaxed and randomly oriented freshwater ice, when deformed slowly at $-10^{\circ} \mathrm{C}$, decreases with increasing grain size, (ii) that the functional relationship between tensile strength of and grain size $d$ is $\sigma_{f}=\sigma_{j}+\mathrm{kd}^{-1 / 2}$, where $\sigma_{j}$ and $\mathrm{k}$ are materials parameters, and $(i i i)$ that the tensile strength of polycrystalline ice is controlled by the propagation in a brittle manner of microcracks nucleated by dislocation interactions.

\section{ACKNOWLEDGEMENT}

This work was funded by the US Army Research office, Contract No. DAA G-29-80-C-0064.

\footnotetext{
*This paper has been published in full: Currier J H, Schulson E M 1982 The tensile strength of ice as a function of grain size. Acta Metallurgica 30(8): 1511-1514

**Now a.t.ARCO 011 \& Gas Co., Dallas, Texas, USA.
} 